\title{
AUTOMATIC REGISTRATION OF APPROXIMATELY LEVELED POINT CLOUDS OF URBAN SCENES
}

\author{
A. Moussa ${ }^{a, b, *}$, N. Elsheimy ${ }^{a}$ \\ ${ }^{a}$ Dept. of Geomatics Engineering, University of Calgary, Calgary, Canada (amelsaye, elsheimy)@ucalgary.ca \\ ${ }^{\mathrm{b}}$ Dept. of Electrical Engineering, Port-Said University, Port-Said, Egypt
}

Commission III, WG III/2

KEY WORDS: Automatic, Registration, Terrestrial, LiDAR, Point Cloud, Urban

\begin{abstract}
:
Registration of point clouds is a necessary step to obtain a complete overview of scanned objects of interest. The majority of the current registration approaches target the general case where a full range of the registration parameters search space is assumed and searched. It is very common in urban objects scanning to have leveled point clouds with small roll and pitch angles and with also a small height differences. For such scenarios the registration search problem can be handled faster to obtain a coarse registration of two point clouds. In this paper, a fully automatic approach is proposed for registration of approximately leveled point clouds. The proposed approach estimates a coarse registration based on three registration parameters and then conducts a fine registration step using iterative closest point approach. The approach has been tested on three data sets of different areas and the achieved registration results validate the significance of the proposed approach.
\end{abstract}

\section{INTRODUCTION}

Terrestrial LiDAR scanning of urban objects typically require many scans from different positions and with different orientations. These multiple scans help to overcome the occlusions encountered by individual scans and offer also a complementary view of the object of interest from different directions. These multiple scans needs to be referenced to a single coordinate frame to form a complete point cloud of the object of interest. This point cloud registration process aims to find the displacement and rotation parameters that transform one point cloud to the reference frame.

In the past two decades, high amount of research effort has been spent towards the registration process. Iterative Closest Point algorithm (ICP) (Besl and McKay, 1992) is an example of the main used algorithms that have been implemented with different variations such as in (Xie et al., 2010) and (Godin et al., 2001). ICP algorithm minimizes the distance between the points of a point cloud and their closest points from the second point cloud. Good initial/coarse registration is needed for the iterative minimization process to converge to an accurate solution.

In (Godin et al., 2001), the points attributes such as intensity and surface curvature are used to assess the compatibility of corresponding points between point clouds and therefore proceed only with the compatible points to obtain a more reliable solution. Geometric Primitive ICP with the RANSAC (GP-ICPR) has been proposed in (Bae and Lichti, 2008) to use geometric attributes such as normal vectors and curvature change to search for the candidate corresponding points, this algorithm still needs initial alignment of the point clouds.

Towards the automation of the registration process, many researches proposed registration algorithms based on special targets to be placed in the scene. These targets should appear in both point clouds where they are detected and matched to offer corresponding points for the registration such as in (Akca, 2003) and (Wang et al., 2014).

(Kang et al., 2009) created panoramic reflectance images form the point clouds and used these images to find the corresponding points needed for registration. With the growing amount of integrated acquisition systems of LiDAR and imagery, many researchers employed this added sensor to help achieve more robust corresponding features between point clouds.

In (Canaz and Habib, 2014), the extracted linear and planar features from both the images and the point clouds are used to find the needed correspondence for registration. The Exterior Orientation Parameters (EOPs) of the images are used to initialize the point clouds registration in (Al-Manasir and Fraser, 2006). (Pandey et al., 2012) proposed mutual information (MI) based approach to fuse the information acquired by the LiDAR and the images towards an automatic registration.

Towards a fully automatic registration, many researchers utilized the expected available features in the point clouds to find the required correspondence for the registration process. (Al-Durgham et al., 2013) proposed a registration approach that uses the extracted 3D linear features from the point clouds and assess hypothesized corresponding linear features to find the matching needed for registration.

(Rabbani et al., 2007) presented an approach where the fitted common objects such as planes, cylinders and spheres from the point clouds are to be matched and integrated into a global registration and modelling process. (Rabbani and van den Heuvel, 2005) adopted a constrained search approach to find the corresponding common objects such as planes and cylinders of the point clouds.

Detection and matching of 3D points of interest have been proposed to automatically find the required points correspondence for the registration process. (Rusu et al., 2008a), (Rusu et al., 2008b), and (Rusu et al., 2009) are examples of $3 \mathrm{D}$ points detection and description algorithms used for the automatic point cloud registration. The Point Feature Histograms (PFH) and Fast Point Feature Histograms (FPFH) are proposed to describe the local geometry around points and therefore can be used in the matching process between the cloud's points.

To reduce the computation load of matching the points between the point clouds, (Barnea and Filin, 2008) exploited the 3D 
rigid body transformation invariant features such as distances to optimize the matching process.

Typically, the registration problem targets the general case of registration and aims to find the registration parameters assuming full range of search space for all the registration parameters. The common scenario of multiple terrestrial scans of urban objects acquire approximately leveled point clouds with small roll and pitch angles' differences and small height difference between the scanners. These small differences can relax the search problem of the registration into two phases where the major three parameters are targeted in the first coarse registration phase and then a fine registration phase can be conducted.

In this paper, an automatic approach is presented where the approximately leveled point clouds are first coarsely registered based on the cross correlation of the horizontal cross section images of the two point clouds, then a fine registration algorithm such as ICP takes place.

The rest of the paper is organized as follows; the proposed methodology is introduced in the next section. Section 3 presents the test's results and discussion, and finally, the conclusions are given in section 4 .

\section{METHODOLOGY}

For the common scenario of multiple scans of urban objects, the scanner is almost leveled at the different scanning locations. This approximate leveling of the scanner leaves small differences of the pitch and roll angles and small difference of height. Only three registration parameters exhibit high values that need to be estimated through a coarse registration algorithm while the other parameters are of small values and can be adjusted later using a traditional fine registration algorithm. The three registration parameters of high values are the two horizontal displacements and the rotation angle around the vertical direction. These three parameters are the required parameters to register two $2 \mathrm{D}$ images.

The proposed algorithm utilizes this search relaxation from six parameters to three parameters to handle the registration process. First, horizontal cross sections of the two point clouds are extracted and gridded into 2D binary images as shown in Figure 1. and Figure 2. The vertical surfaces of the urban objects exhibit straight linear features in the created cross section images which help to find a reliable registration between the images. These two created images are to be registered through finding three registration parameters. The 2D crosscorrelation between the first image and the rotated versions of the second image is computed. The highest $2 \mathrm{D}$ cross-correlation value indicates the highest matching between the two images. Figure 3 exhibits the maximum 2D cross-correlation at different rotation angles.

The rotated image, at which this cross-correlation value is found, specifies the estimated rotation angle around the vertical direction between the two point clouds. The horizontal displacement between the two point clouds can be calculated using the position of the highest $2 \mathrm{D}$ cross correlation value.

Figure 4 shows the registered cross section images using the estimated horizontal displacement and rotation angle.

The second point cloud is coarsely registered with the first point cloud by transforming it using the three estimated registration parameters. Finally the coarsely registered point clouds are fine registered using ICP algorithm.

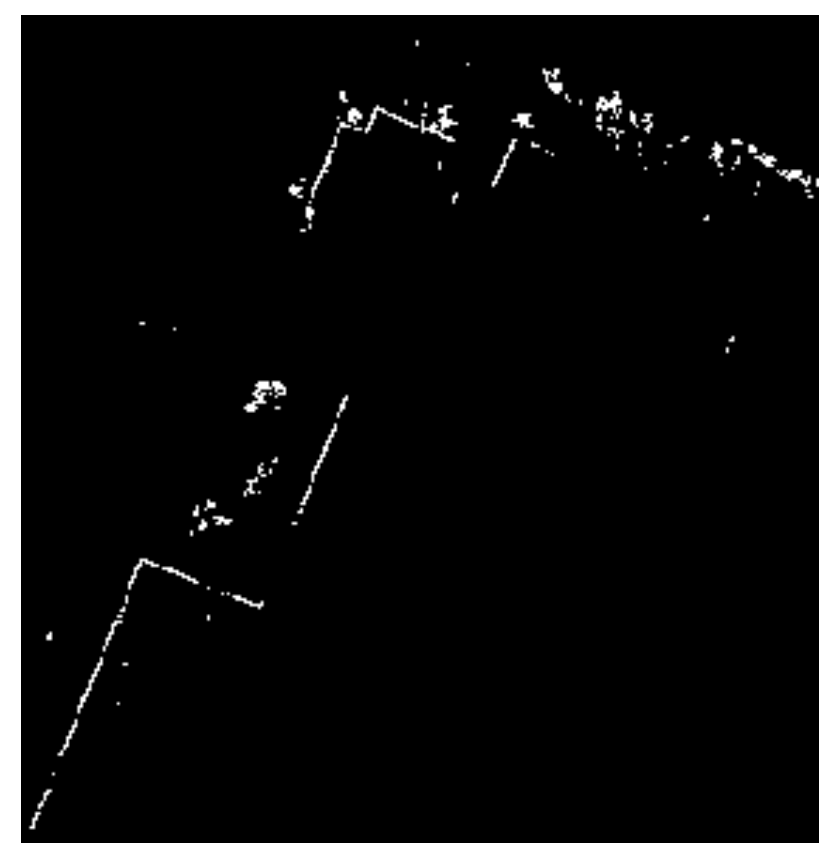

Figure 1. Cross section image of first point cloud

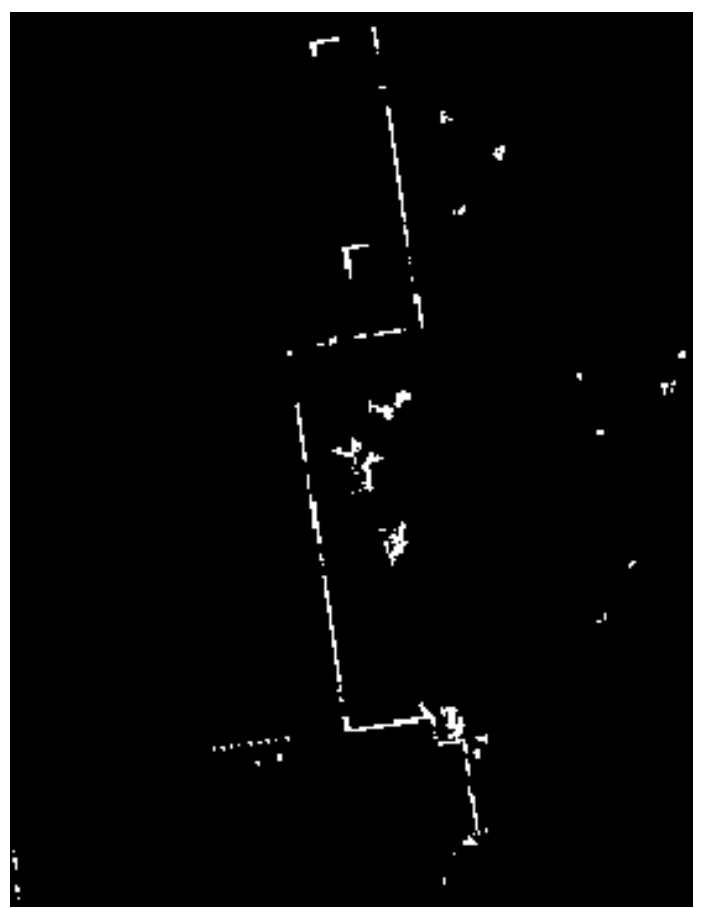

Figure 2. Cross section image of second point cloud at zero rotation angle 


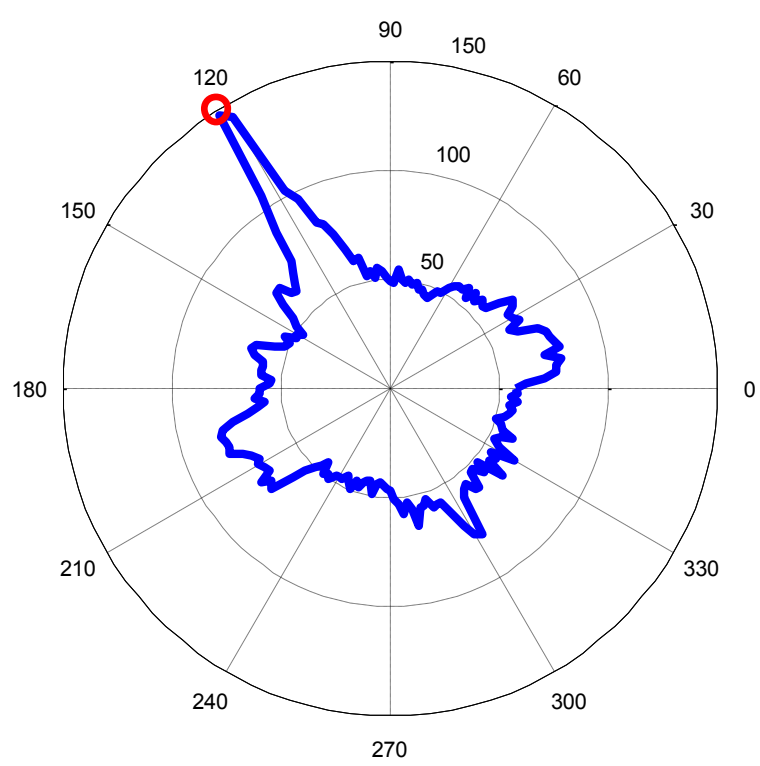

Figure 3. Maximum cross-correlation at the different rotation angles

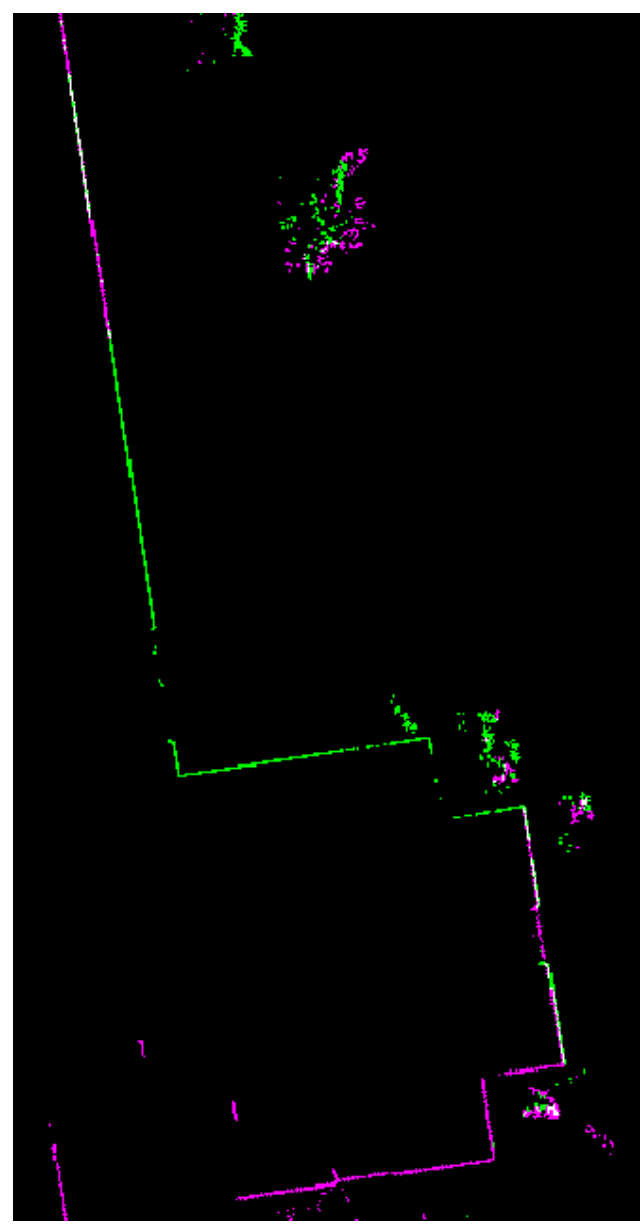

Figure 4. Zoomed registered cross section images using the estimated horizontal displacement and rotation angle

The methodology details are summarized in the following algorithm
Algorithm 1: Automatic registration

i. For each point cloud, find the points at the scanner height and within a tolerance.

ii. Project vertically the found points of the first point cloud as white pixels on a black image.

iii. For angle $=-180$ to 180 with $2^{\circ}$ degrees increments, rotate the found points of the second point cloud and create a corresponding image as in step (ii) for each angle.

iv. Compute the 2D cross-correlation between the image of first point cloud and the images computed in step (iii)

v. Find the maximum value of cross-correlation across all the images and estimate rotation angle $=$ the rotation of the image at which the maximum value was found,

vi. Repeat steps (iii-v) with smaller grid cell size and using finer angle step in a small range around the previously estimated rotation angle.

vii. Estimate rotation angle $=$ the rotation of the image at which the maximum value was found in step (vi), and the horizontal displacement is computed using the position of the maximum value in the corresponding image.

viii. Transform the second point cloud using the estimated horizontal displacement and rotation angle.

ix. Conduct ICP fine registration between the first point cloud and the transformed second point cloud.

To speed up the coarse registration process, first, this whole process is performed using a large grid cell size and also scans the angles from $-180^{\circ}$ to $180^{\circ}$ using a relatively large angle step size.

This large grid cell size and angle step reduces the number and the size of the cross section images and therefore reduces greatly the processing time.

After the completion of this first stage, the process is repeated again using a finer grid cell size and finer angle step around the estimated rotation angle in the first stage. This second stage scans only the angles in a small range around the previously estimated rotation angle.

These two stages help to reduce the computation burden and to maintain at the same time the needed accuracy for the last phase where the ICP approach takes place.

\section{RESULTS AND DISCUSSION}

The proposed approach has been tested on three data sets of terrestrial laser scanning. In the first dataset, two terrestrial laser scans of the Rozsa center, a conference center at the University of Calgary, have been registered using the proposed approach. The scans were collected using a Trimble terrestrial laser scanner (GS200) with a maximum range of $200 \mathrm{~m}$ and a resolution up to 32 milliradians (or $3 \mathrm{~mm}$ at $100 \mathrm{~m}$ ). 


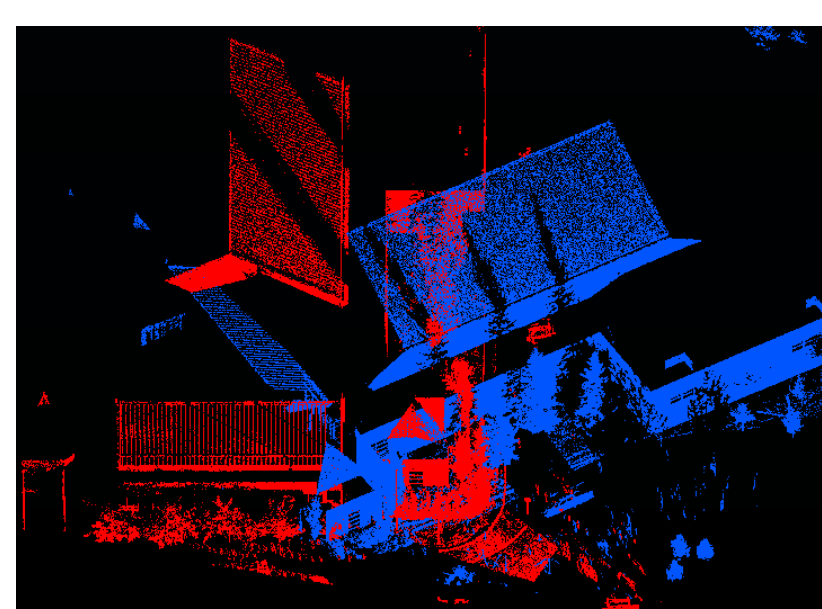

Figure 5. Two subsampled point clouds of Rozsa center before registration

Figure 5 shows the two point clouds of Rozsa center before registration. These two point clouds have been sub sampled with average points spacing of around $5 \mathrm{~cm}$. Figure 6 shows the two point clouds of Rozsa center after registration. The average point to point distance between the overlapped areas of the registered point clouds is around $4.8 \mathrm{~cm}$.

A tolerance of $0.1 \mathrm{~m}$ has been used to find the cross section points, and a grid cell size of $30 \mathrm{~cm}$ has been selected during images creation the grid cell size has been reduced to $5 \mathrm{~cm}$ in the fine image registration stage.

Despite the random tree points and the incomplete line segments of the two cross section images as depicted in Figure 1 and Figure 2, the image registration was successfully achieved using the proposed approach as depicted in Figure 4 where the violet and green colors indicate the points of the first and the second cross section images respectively.

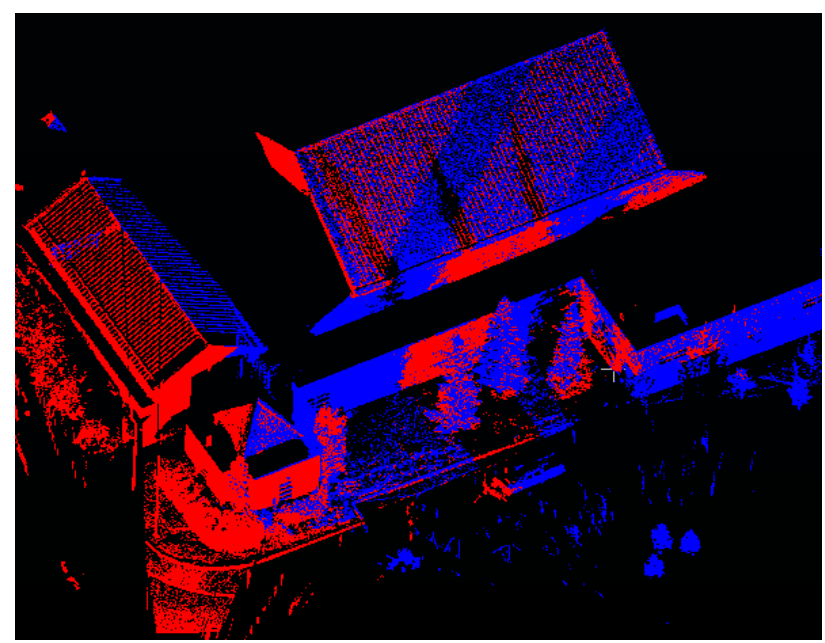

Figure 6. The two sub sampled point clouds of Rozsa center after registration

In the second dataset, two terrestrial laser scans of the Yamnuska hall, a residence building at the University of Calgary, have been registered using the proposed approach. The scans were collected by Leica TCR 803 TLS with 1 inch of display resolution.

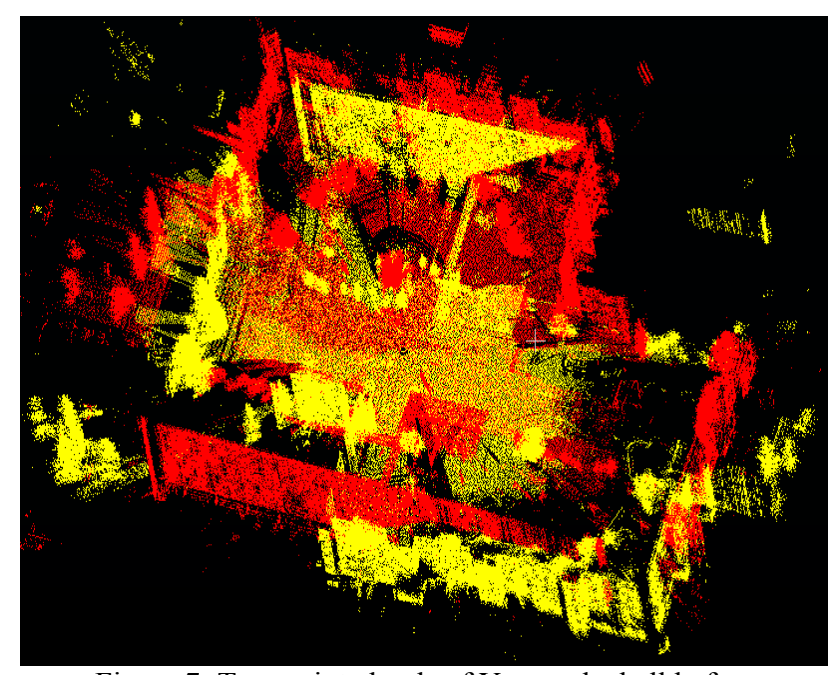

Figure 7. Two point clouds of Yamnuska hall before registration

Figure 7 shows the two point clouds of Yamnuska hall before registration. The two scan are almost in opposite directions with many trees across the scene. Figure 8 shows the two point clouds of Yamnuska hall after registration. The average point to point distance between the overlapped areas of the registered point clouds is around $6 \mathrm{~cm}$.

The same tolerance of $0.1 \mathrm{~m}$ and the same grid cell sizes of 30 and $5 \mathrm{~cm}$ have been employed in the cross section point's selection and the images creation of this data set. The straight line segments of the cross sections helped to compensate the effect of the random positions of the trees cross section points on the image registration.

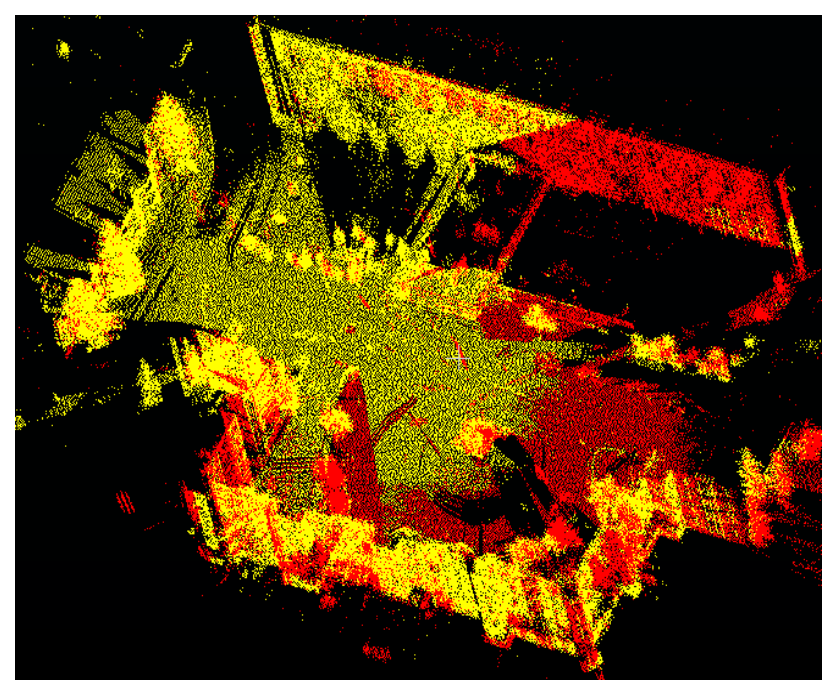

Figure 8. The two point clouds of Yamnuska hall after registration

The third dataset is a collection of 12 terrestrial laser scans offered by the Institute of Cartography and Geoinformatics, Leibniz Universität Hannover. The data set is published at webpage: http://www.ikg.uni-hannover.de. The twelve scans were collected at 2006 with approximately 2.5 million points in each scan. Figure 9 shows the twelve point clouds of Holzmarkt dataset before registration where each point cloud has been given a random color. 
These twelve scans have been registered using the proposed approach in a successive fashion. The homogenous transformation matrix $R_{i, i+1}$ between each two successive point clouds $\mathrm{P}_{\mathrm{i}}, \mathrm{P}_{\mathrm{i}+1}$ is estimated using the proposed approach. Then these relative transformation matrices are used to find the transformation matrices between the scans and the first (reference) scan to register all these scan to a single reference frame.

The same tolerance of $0.1 \mathrm{~m}$ and the same grid cell sizes of 30 and $5 \mathrm{~cm}$ have been employed in the cross section point's selection and the images creation of this data set.

Figure 10 depicts the twelve point clouds of Holzmarkt dataset after registration with height based colouring. The average point to point distance between the overlapped areas of the successive registered point clouds is between 3 and $6 \mathrm{~cm}$.

Despite the accumulated transformation between the first reference scan and the twelfth scan, the final registration result does not exhibit a significant drift over the scans, which proves the effectiveness of the proposed approach.

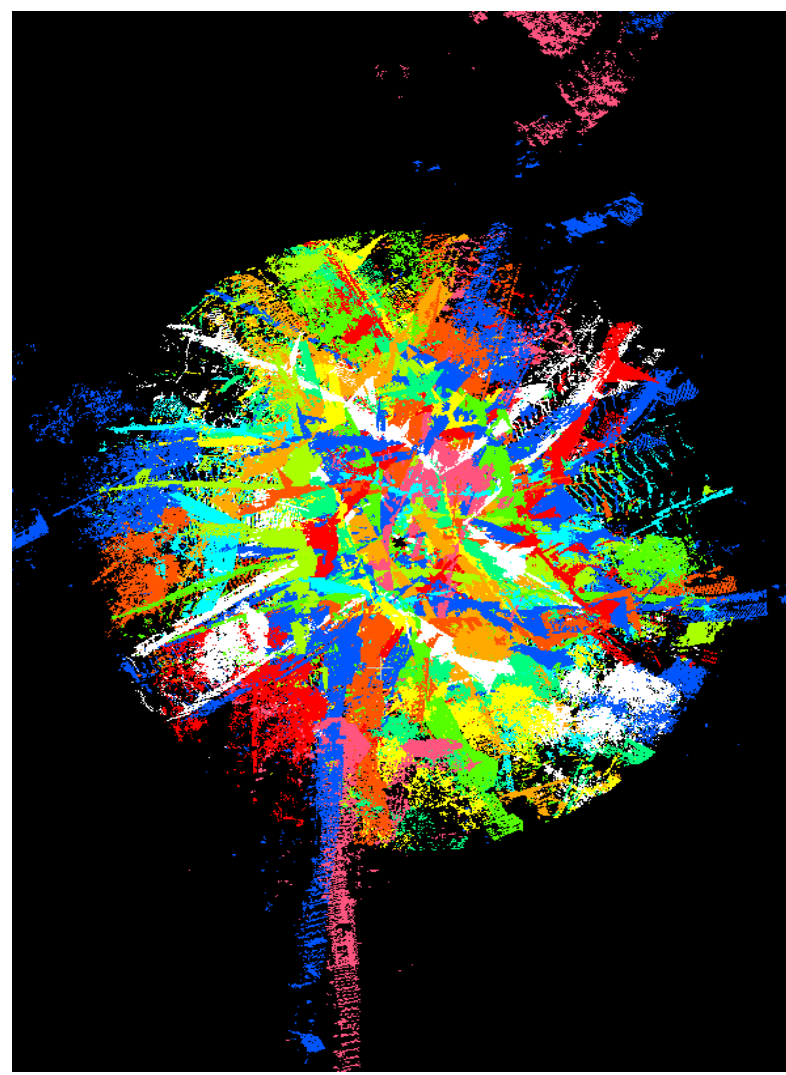

Figure 9. Twelve point clouds of Holzmarkt dataset before registration (each point cloud with a random color)

The needed computation time throughout the tested data sets varies based on the extent of the scan where some of the scans in the data sets extend for $200 \mathrm{~m}$. For the 13 tested (two scans) registrations, the total computation time of the coarse registration between two scans varies from 49s to 266s. The processing has been performed using Matlab software with an Intel i7-4702MQ processor.

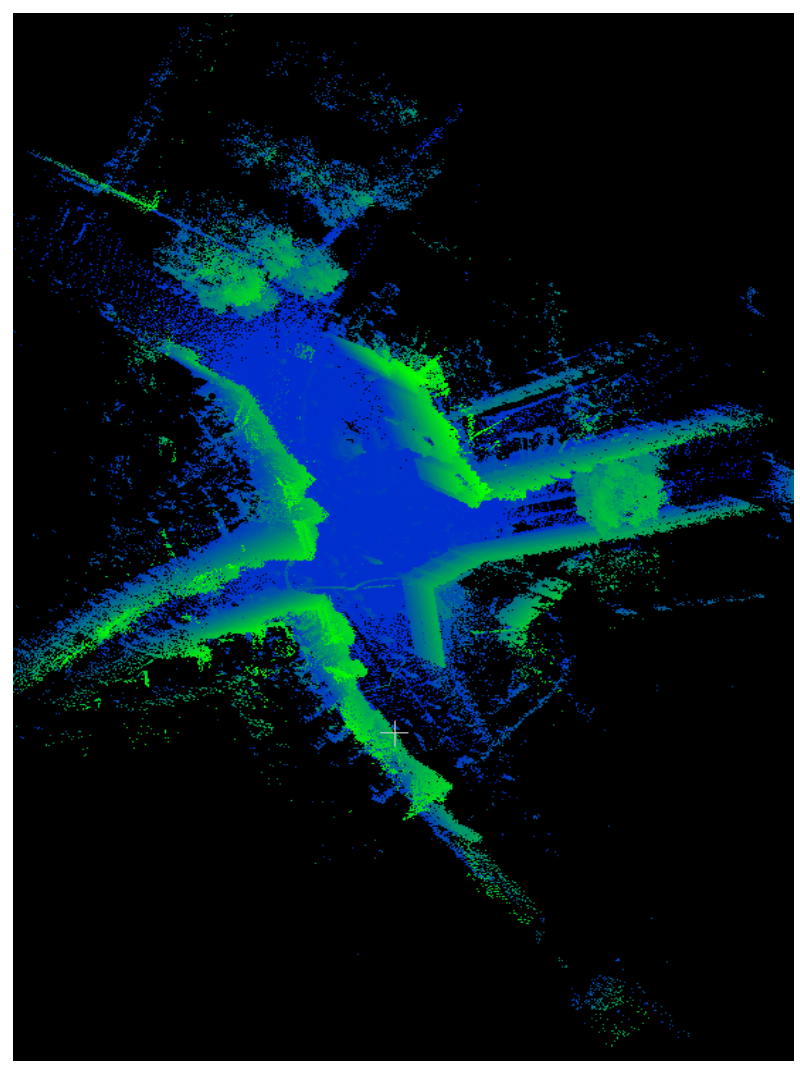

Figure 10. the twelve point clouds of Holzmarkt dataset after registration with height based colouring

\section{CONCLUSIONS}

In this paper, an automatic registration approach for approximately leveled point clouds of urban scenes is proposed. The approximate leveling of the scans constrains three of the six registration parameters into small values. The other three registration parameters, two horizontal displacements and a rotation angle around the vertical direction are estimated in the proposed approach as image registration parameters. Two cross section images of the point clouds are created and registered to find these three registration parameters. After this coarse registration takes place, a fine registration phase is conducted using ICP approach. The proposed algorithm deals with a very small subset of the cloud points and utilizes the well-established 2D cross correlation operation which can efficiently run to obtain a fast and accurate registration result. The proposed method has been implemented and tested on three data sets of terrestrial laser scanning. The registration results of the data sets prove the significance of the proposed approach.

\section{ACKNOWLEDGEMENTS}

This work was supported in part by Dr. Naser El-Sheimy research funds.

\section{REFERENCES}

Akca, D., 2003. Full automatic registration of laser scanner point clouds. Optical 3-D Measurement Techniques VI, Zurich, Switzerland, pp. 330-337. 
Al-Durgham, K., Habib, A., Kwak, E., 2013. RANSAC approach for automated registration of terrestrial laser scans using linear features. In: ISPRS Annals of Photogrammetry, Remote Sensing and Spatial Information Sciences. ISPRS Workshop Laser Scanning 2013. Antalya, Turkey, Volume II5/W2.

Al-Manasir, K. and Fraser, C.S., 2006. Registration of terrestrial laser scanner data using imagery. The Photogrammetric Record 21(115), pp. 255-268.

Bae, K.H. and Lichti, D.D., 2008. A method for automated registration of unorganised point clouds. ISPRS Journal of Photogrammetry and Remote Sensing 63(1), pp. 36-54.

Barnea, S. and Filin, S., 2008. Keypoint based autonomous registration of terrestrial laser point-clouds. ISPRS Journal of Photogrammetry and Remote Sensing 63(1), pp. 19-35.

Besl, P. J. and McKay, N.D., 1992. Method for registration of 3-D shapes. IEEE Transactions on Pattern Analysis and Machine Intelligence, 14(2), pp. 239-256.

Canaz, S. and Habib, A., 2014. Photogrammetric features for the registration of terrestrial laser scans with minimum overlap. Journal of Geodesy and Geoinformation 2(1), pp. 1-8.

Godin, G., Laurendeau, D., Bergevin, R., 2001. A method for the registration of attributed range images, in: Third International Conference on 3D Digital Imaging and Modeling, Québec, Canada, pp. 179-186.

Kang, Z., Li, J., Zhang, L., Zhao, Q., Zlatanova, S., 2009. Automatic registration of terrestrial laser scanning point clouds using panoramic reflectance images. Sensors. 9(4), pp. 26212646.

Pandey, G., , McBride, J. R., Savarese, S., Eustice, R.M., 2012. Toward mutual information based automatic registration of $3 \mathrm{D}$ point clouds. Proceedings of IEEE/RSJ International Conference on Intelligent Robots and Systems, Algarve, Portugal, pp. 2698-2704.

Rabbani, T., Dijkman, S., van den Heuvel, F., Vosselman, G., 2007. An integrated approach for modelling and global registration of point clouds. ISPRS Journal of Photogrammetry and Remote Sensing. 61(6), pp. 355-370

Rabbani, T., van den Heuvel, F., 2005. Automatic point cloud registration using constrained search for corresponding objects. Proceedings of 7 th Conference on Optical 3-D Measurement Techniques. Vienna, Austria, Vol. 1, pp. 177-186.

Rusu, R.B., Blodow, N., Beetz, M., 2009. Fast Point Feature Histograms (FPFH) for 3D Registration, in: Proceedings of the IEEE International Conference on Robotics and Automation (ICRA), pp. 3212-3217.

Rusu, R.B., Blodow, N., Marton, Z.C., Beetz, M., 2008a. Aligning point cloud views using persistent feature histograms, in: Proceedings of the 21st IEEE/RSJ International Conference on Intelligent Robots and Systems, IROS, Nice, France, pp. 3384-3391.

Rusu, R.B., Marton, Z.C., Blodow, N., Beetz, M., 2008b. Learning informative point classes for the acquisition of object model maps. in Proceedings of the 10th International Conference on Control, Automation, Robotics and Vision (ICARCV), Hanoi, Vietnam.

Wang, Y., Shi, H., Zhang, Y., Zhang, D., 2014. Automatic registration of laser point cloud using precisely located sphere targets. Journal of Applied Remote Sensing, 8(1).

Xie, Z., Xu, S., Li, X., 2010. A high-accuracy method for fine registration of overlapping point clouds. Image and Vision Computing 28(4), pp. 563-570. 